\title{
The LINC01186 suppresses cell proliferation and invasion ability in papillary thyroid carcinoma
}

\author{
NANPENG WANG, HAISONG DUAN, CHEN ZHANG, YAN ZHOU and RONGJUN GAO
}

Department of Thyroid Surgery, Affiliated Hospital of Guizhou Medical University, Guiyang, Guizhou 550004, P.R. China

Received November 4, 2017; Accepted June 14, 2018

DOI: $10.3892 / 01.2018 .9349$

\begin{abstract}
Papillary thyroid carcinoma (PTC) is the most common thyroid malignancy. Long non coding RNAs (lncRNAs) have been involved in regulating tumor progression including PTC. In the present study, we demonstrated that long non coding RNA LINC01186 was significantly downregulated in PTC tissue samples compared with adjacent normal tissue samples in patients. LINC01186 expression was also found to be higher in PTC cells. Lower LINC01186 expression was associated with lymph node metastasis of PTC patients. Functionally, LINC01186 overexpression significantly suppressed cell proliferation, cell colony formation and cell invasion ability in TPC-1 and IHH-4 cells. In addition, we demonstrated that LINC01186 overexpression inhibited large tumor suppressor kinase 1 (LATS1)/YY1 associated protein 1 (YAP) signaling by reducing YAP1 expression, while increasing LATS1 expression in TPC-1 and IHH-4 cells. Collectively, our data suggested that LINC01186 may serve as a potential target for therapy in thyroid carcinoma.
\end{abstract}

\section{Introduction}

Thyroid cancer is rare, but it is the most frequent endocrine malignancy. Thyroid cancer is divided into four types including papillary, follicular, medullary, and anaplastic thyroid cancer $(1,2)$. Papillary thyroid carcinoma (PTC) accounts for approximately more than $85 \%$ of all thyroid cancers. Although PTC exhibits a satisfactory outcome after surgery or radioiodine, however, approximately $15 \%$ of PTC patients who have relapsed and frequently distant metastases present poor prognosis $(3,4)$. Thus, to investigate underling molecular mechanisms involved in carcinogenesis of PTC was urgent.

Correspondence to: Dr Nanpeng Wang, Department of Thyroid Surgery, Affiliated Hospital of Guizhou Medical University, 28 Guiyi Street, Guiyang, Guizhou 550004, P.R. China

E-mail: ke62339260@163.com

Key words: papillary thyroid carcinoma, long noncoding RNA, LINC01186, YAP1, LATS1
Long non coding RNAs (lncRNAs) have been identified as crucial regulators in many biological processes, such as the cell cycle, cell apoptosis, senescence, cell differentiation and tumorigenesis $(5,6)$. Abnormal expression of lncRNAs was involved in PTC development and progression. For example, PVT1 (7), HOTAIR (8), and MEG3 (9) and so on, potentially function as oncogenes or tumor suppressors of PTC. LINC01186 was reported to acts as a tumor suppressor in lung cancer and inhibits migration and invasion through Epithelial-Mesenchymal-Transition of lung cancer (10). However, little was known for LINC01186 in PTC development and progression.

In the study, we demonstrated that LINC01186 was significantly downregulated in PTC tissues compared to adjacent normal tissues. LINC01186 overexpression suppressed cell proliferation and invasion ability. Moreover, we demonstrated that LINC01186 overexpression suppressed thelarge tumor suppressor kinase (LATS1)/ YY1 associated protein 1 (YAP1) signaling in PTC cells. Thus, our data suggested that LINC01186 may serve as a target for therapy in PTC.

\section{Materials and methods}

Tissue samples. A total of 65 cases PTC tissue sample and adjacent normal tissue sample were obtained between April 2012 and January 2014 at Department of Thyroid Surgery, Affiliated Hospital of Guizhou Medical University (Guizhou, China). All patients (22 male and 43 female; age range, 23-76 years; mean age, 45 years) were enrolled in the study did not receive any preoperative therapies including radiotherapy, chemotherapy and levothyroxine, prior to the thyroidectomy. All of tissue samples were immediately frozen in liquid nitrogen and stored at $-80^{\circ} \mathrm{C}$ following operation until further RNA extraction. The study protocol was approved by the Ethics Committee of Affiliated Hospital of Guizhou Medical University and written informed consent was obtained from all of patients.

Cell lines culture. Three human PTC cell lines (TPC-1, K1 and IHH-4) and a human thyroid epithelial cell line Nthy-ori3-1 were purchased from the Cell Bank of the Chinese Academy of Sciences (Shanghai, China). Although K1 cells are contaminated with GLAG-66 (11), the resulting phenotypic and genotypic differences between K1 and GLAG-66 cells were considered unlikely to affect our study. The PTC cells 
were supplemented with Dulbecco's modified Eagle's medium (DMEM) containing 10\% fetal bovine serum (FBS; both Gibco; Thermo Fisher Scientific Inc., Waltham, MA, USA). Cells were cultured at $37^{\circ} \mathrm{C}$ in humidified air with $5 \% \mathrm{CO}_{2}$.

Plasmid construction. The full-length LINC01186 sequences were chemically synthesized and cloned into the pcDNA3.1 plasmid by Guangzhou RiboBio Co., Ltd. (Guangzhou, China). The pcDNA3.1-LINC01186 plasmid was transfected into TPC-1 and IHH-4 cells using Lipofectamine 2000 (Invitrogen; Thermo Fisher Scientific, Inc.).

RNA extraction and reverse transcription-quantitative $P C R$ (RT- $q P C R)$. Total RNA was extracted using Trizol reagent (Invitrogen; Thermo Fisher Scientific, Inc.) following the manufacturer's instructions. LINC01186 cDNA was reverse-transcribed using Prime Script TM RT-PCR kit and were detected using SYBR Premix Ex TaqTM (both Takala, Dalian, China) under an ABI 7500 Fast Sequence Detection System (Applied Biosystems, Foster City, CA, USA). The GAPDH mRNA expression was used as internal control. The primer sequences were as follows: LINC01186-forward, 5'-CTA AAACGGGAAAGATGCCTTC-3' and reverse, 5'-TCAAGA TTAGGGAACATACTGGC-3'; GAPDH forward, 5'-GGA GCGAGATCCCTCCAAAAT-3' and reverse, 5'-GGCTGT TGTCATACTTCTCATGG-3'. Data was quantified using the $2^{-\Delta \Delta C q}$ method (12).

Cell proliferation assay. PTC cells (3,000 cells per well) were seeded in 96-well plates were and cultured for $24 \mathrm{~h}$. Then, cells were transfected with si-NC and si-LINC01186 and cultured at $37^{\circ} \mathrm{C}$ in humidified air with $5 \% \mathrm{CO}_{2}$. Cell proliferation ability was measured by using the Cell Counting kit-8 (CCK-8; Dojindo, Kumamoto, Japan) at 12, 24, 48, and $72 \mathrm{~h}$. The absorbance was at $450 \mathrm{~nm}$ under a microplate spectrophotometer (Thermo Labsystems, Vantaa, Finland).

Cell colony formation assay. PTC cells (3,000 cells per well) were seeded in 6-well plates were and cultured for $24 \mathrm{~h}$. Then, cells were transfected with si-NC and si-LINC01186 and cultured at $37^{\circ} \mathrm{C}$ in humidified air with $5 \% \mathrm{CO}_{2}$ for 7 days. Cell colonies were fixed with $4 \%$ paraformaldehyde, stained with $0.1 \%$ crystal violet. Cells colonies were calculated under a microscope (Olympus, Tokyo, Japan).

Cell invasion assay. Cell invasion assays were performed using transwell chambers with membrane pore size of $8.0 \mu \mathrm{m}$ (Corning Inc., Union City, CA, USA). Transfected cells $\left(1 \times 10^{5}\right.$ cells $)$ were added into the upper chamber, while the lower chamber contained DMEM medium with $10 \%$ FBS After cell culture for $48 \mathrm{~h}$, cells in the lower chamber were fixed with $4 \%$ paraformaldehyde and stained with $0.1 \%$ crystal violet. Invasive cells were calculated under a microscope (Olympus) in five random fields.

Western blot assay. Total proteins were extracted from transfecting cells using RIPA lysis buffer (Pierce, Rockford, IL, USA). After centrifugation at $14,000 \mathrm{~g}, 4^{\circ} \mathrm{C}$ for $15 \mathrm{~min}$, the supernatants were collected. The protein concentration was detected using a BCA protein assay kit (Thermo Fisher Scientific Inc.). Equal protein was separated at sodium dodecyl sulfate-polyacrylamide gel electrophoresis (SDS-PAGE), and then transferred to PVDF membranes (Millipore, Billerica MA, USA). Then, the membranes were blocked with 5\% non-fat milk and incubated with primary antibody: Anti-YAP1 $(1: 1,000)$, anti-LATS1 $(1: 1,000)$ and GAPDH (1:1,000 all Cell Signaling Technology, Inc., Danvers, MA, USA). After incubation with the appropriate horseradish peroxidase (HRP)-conjugated secondary antibody for $2 \mathrm{~h}$, the blots were detected using an enhanced chemiluminescence detection system (Amersham, Little Chalfont, UK). GAPDH were used as an internal control.

Statistical analysis. The SPSS 20.0 software system (IBM Corp., Armonk, NY, USA) was used for statistical analyses. The results are showed as the mean $\pm \mathrm{SD}$ (standard deviation) from at least three independent experiments. The chi-square test was applied to examine the relationship between LINC01186 expression and clinicopathological characteristics. Significant differences between two groups were compared using two-tailed Student's t-tests. Significant differences among multiple groups were performed using one-way analysis of variance followed by a Bonferroni post hoc test. $\mathrm{P}<0.05$ was considered to indicate a statistically significant difference.

\section{Results}

Expression of LINC01186 is significantly downregulated in PTC tissues and cells. To explore the clinical role of LINC01186 in PTC, we examined the expression of LINC01186 in PTC tissue samples and adjacent normal tissue samples using RT-qPCR. The results showed that LINC01186 expression levels were notably downregulated in PTC tissues compared to adjacent normal tissues $(\mathrm{P}<0.05$; Fig. $1 \mathrm{~A})$. The median expression of LINC01186 was used as a cut-off value to divide 65 cases into two groups (lower or higher group). We found that lower LINC01186 expression was associated with lymph node metastasis of PTC patients $(\mathrm{P}<0.05$; Table I). Moreover, a decreased expression of LINC01186 was also observed in three different PTC cell lines compared to human thyroid epithelial cell line Nthy-ori3-1 ( $\mathrm{P}<0.05$; Fig. 1B). Collectively, all the results indicated that LINC01186 is downregulated in PTC.

LINC01186 inhibits cell proliferation and migration of PTC in vitro. To explore the biological role of LINC01186 in PTC progression, we performed gain-function assays by transfection with pcDNA3.1-LINC01186 plasmids into TPC-1 and IHH-4 cells. The efficiency of LINC01186 overexpression by pcDNA3.1-LINC01186 plasmid was confirmed by RT-qPCR and shown in Fig. 1C $(\mathrm{P}<0.05)$. CCK8 cell proliferation assays demonstrated that enhanced expression of LINC01186 suppressed the proliferation ability of TPC- 1 and IHH- 4 cells compared with the control cells transfected with empty vector $(\mathrm{P}<0.05$; Fig. 2A and B). Cell colony formed number was reduced when LINC01186 was overexpressed after transfected with pcDNA3.1-LINC01186 plasmids into TPC-1 and IHH-4 cells compared with the control cells transfected with empty vector $(\mathrm{P}<0.05$; Fig. $2 \mathrm{C}$ and $\mathrm{D})$. Moreover, we demonstrated 

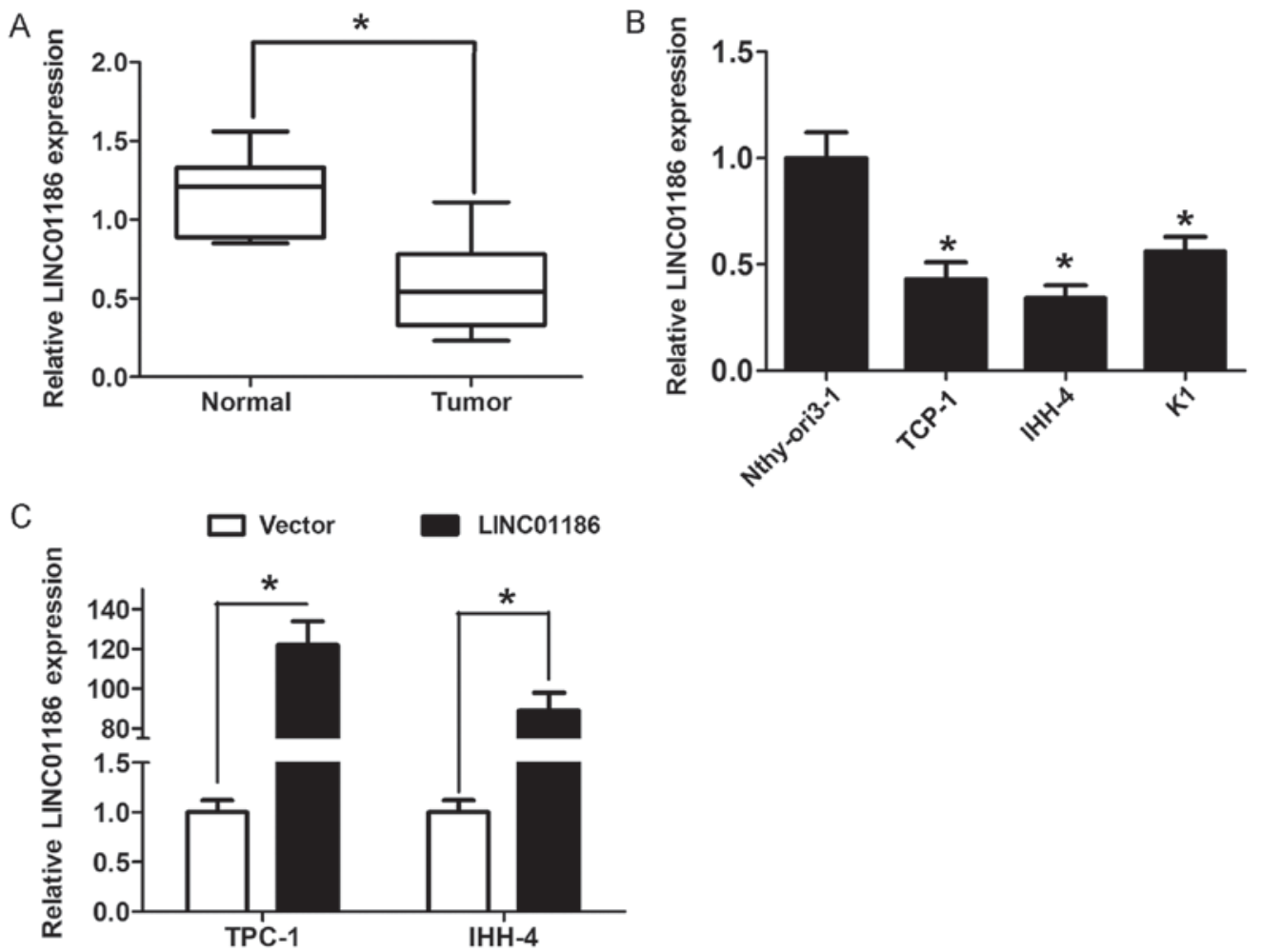

Figure 1. LINC01186 expression was downregulated in PTC tissues and cells. (A) LINC01186 expression was detected in 65 cases of PTC tissue samples and adjacent normal tissue samples by using RT-qPCR. (B) LINC01186 expression was detected in three human PTC cell lines (TPC-1, K1 and IHH-4) and a human thyroid epithelial cell line Nthy-ori3-1by using RT-qPCR. (C) LINC01186 expression was detected after TPC-1 and IHH-4 cells were transfected with pcDNA3.1-vector or pcDNA3.1-LINC01186 using RT-qPCR. * $\mathrm{P}<0.05$. PTC, papillary thyroid carcinoma

that upregulated expression of LINC01186 suppressed the cell invasion ability of TPC-1 and IHH-4 cells compared with the control cells transfected with empty vector $(\mathrm{P}<0.05$, Fig. $3 \mathrm{~A}$ and B). Thus, these results indicated that LINC01186 inhibited cell proliferation and invasion of PTC in vitro.

LINC01186 inhibits LATS1/YAP signaling pathway in PTC cells. LATS1/YAP signaling pathway executes important function in PTC progression. High expression of Yes-activated protein-1 in PTC correlates with poor prognosis (13). In thyroid cancer cell lines, Yes-associated protein 1 promotes papillary thyroid cancer cell proliferation by activating the ERK/MAPK signaling pathway (14). In the study, we demonstrated that upregulated expression of LINC01186 suppressed the expression levels of YAP1 and upregualted the LATS1 in TPC-1 and IHH-4 cells compared with the control cells transfected with empty vector $(\mathrm{P}<0.05$; Fig. $3 \mathrm{C}$ and $\mathrm{D})$. Moreover, we also detected the mRNA expression of YAP downstream targeted gene CTGF and Cyclin A. The results showed that upregulated of LINC01186 expression suppressed the mRNA expression levels of CTGF and Cyclin A in TPC-1 and IHH-4 cells (Fig. 4A and B). Thus, these results indicated that LINC01186 inhibited LATS1/YAP signaling pathway in PTC cells.

\section{Discussion}

LncRNAs have been recently found to be dysregulated and act as crucial regulators in the progression of thyroid cancer. For instance, the lncRNA n340790 accelerates carcinogenesis of thyroid cancer by regulating miR-1254 (15). Knockdown of IncRNA-PANDAR suppresses the proliferation, cell cycle and promotes apoptosis in thyroid cancer cells (16). Long non-coding RNA ANRIL promotes the invasion and metastasis of thyroid cancer cells through TGF- $\beta / \mathrm{Smad}$ signaling pathway (17). Overexpression of long intergenic noncoding RNA LINC00312 inhibits the invasion and migration of thyroid cancer cells by downregulating microRNA-197-3p (18). In the study, we demonstrated that long noncoding RNA LINC01186 was significantly downregulated in PTC tissues compared to adjacent normal tissues. Furthermore, in vitro, CCK8 cell proliferation and cell colony formation showed that LINC01186 overexpression suppressed cell proliferation ability. Meanwhile, cell invasion was also been inhibited by overexpression of LINC01186 in thyroid carcinoma cells. These results indicated that LINC01186 suppressed cell proliferation and invasion.

Many studies have demonstrated YAP, core downstream target of Hippo signaling pathway, is altered in different malignant tumors including thyroid carcinoma (19). In thyroid carcinoma, Ugolini et al reported that YAP-1 is overexpressed in papillary and anaplastic thyroid cancers and associated with poor prognosis of thyroid cancer (13). Liu et al showed that YAP expression positively correlated with TNM stage and lymph node metastasis and knockdown of YAP inhibited cell proliferation, migration and invasion in thyroid carcinoma cells (20). Yes-associated protein 1 promotes papillary thyroid cancer cell proliferation by activating the ERK/MAPK signaling pathway (14). In the study, 
Table I. Association between LINC01186 expression and clinicopathological factors in PTC patients.

\begin{tabular}{|c|c|c|c|c|}
\hline \multirow[b]{2}{*}{ Clinicopathological factors } & \multicolumn{3}{|c|}{ LINC01186 expression } & \multirow[b]{2}{*}{ P-value } \\
\hline & Patients $(n=65)$ & Lower $(n=32)$ & Higher $(n=33)$ & \\
\hline Age, years & & & & 0.821 \\
\hline$\leq 55$ & 50 & 25 & 25 & \\
\hline$>55$ & 15 & 7 & 8 & \\
\hline Sex & & & & 0.261 \\
\hline Male & 22 & 10 & 12 & \\
\hline Female & 43 & 22 & 21 & \\
\hline Tumor size, cm & & & & 0.511 \\
\hline$\leq 2$ & 31 & 13 & 18 & \\
\hline$>2$ & 34 & 19 & 15 & \\
\hline Lymph node metastasis & & & & $0.009^{\mathrm{a}}$ \\
\hline Negative & 35 & 12 & 23 & \\
\hline Positive & 30 & 20 & 10 & \\
\hline Tumor location & & & & 0.494 \\
\hline Unilateral & 36 & 20 & 16 & \\
\hline Bilateral & 18 & 7 & 11 & \\
\hline Multifocality & 11 & 5 & 6 & \\
\hline TNM stage & & & & 0.223 \\
\hline I & 33 & 13 & 20 & \\
\hline II & 20 & 11 & 9 & \\
\hline III-IV & 12 & 8 & 4 & \\
\hline
\end{tabular}

${ }^{\mathrm{a}} \mathrm{P}<0.05$. TNM, tumor node metastasis; PTC, papillary thyroid carcinoma.
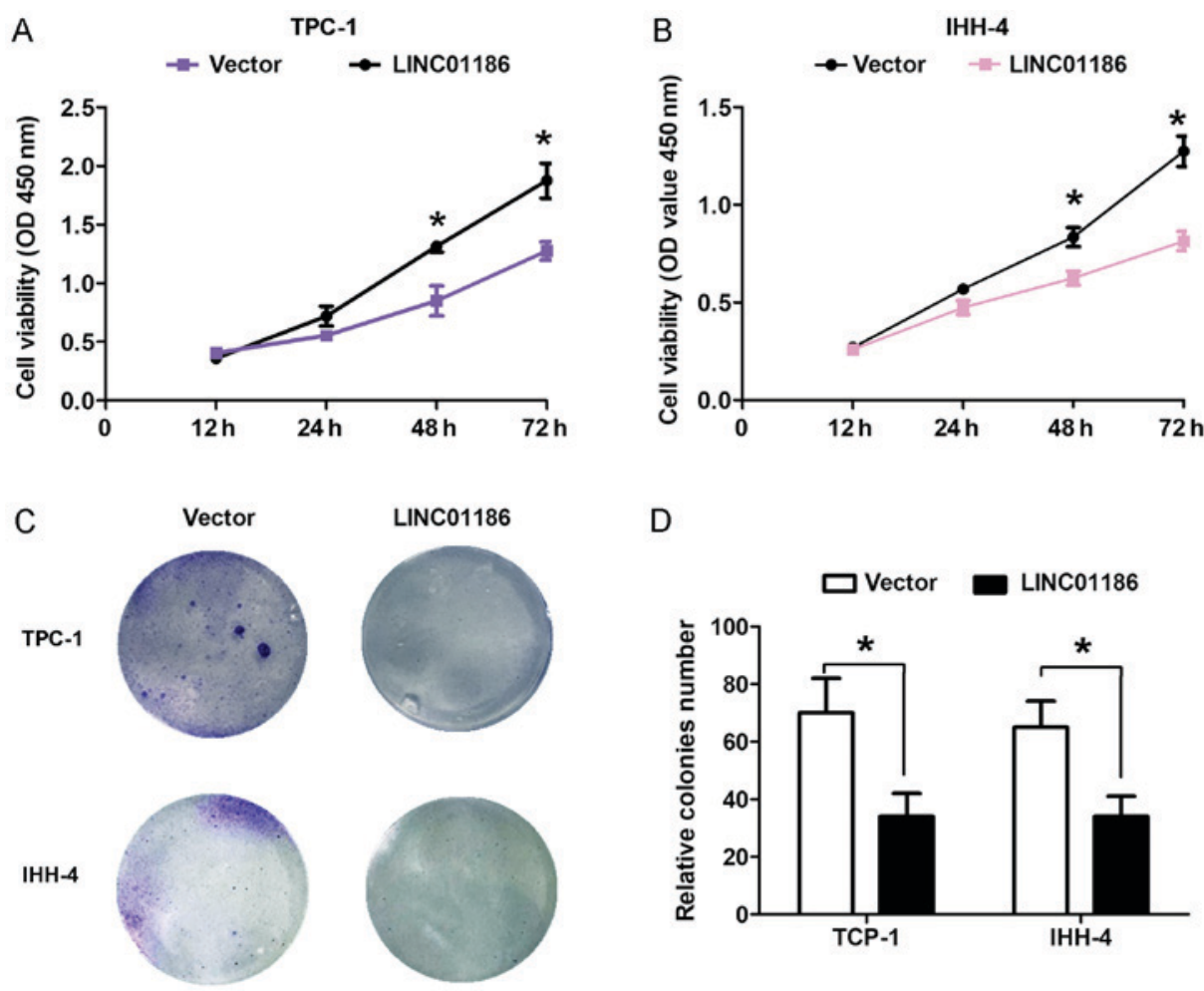

D

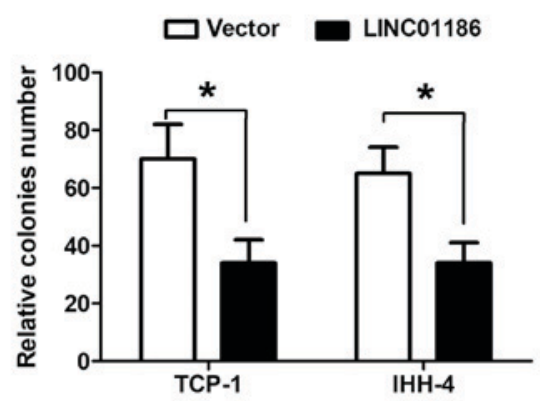

Figure 2. LINC01186 overexpression inhibited cell proliferation of PTC in vitro. CCK8 cell proliferation assays were used to detect cell proliferation ability after (A) TPC-1 and (B) IHH-4 cells were transfected with pcDNA3.1-vector and pcDNA3.1-LINC01186 plasmid. (C) Representative image and (D) quantification of cell colony formation assays used to detect cell proliferation ability after TPC-1 and IHH-4 cells were transfected with pcDNA3.1-vector and pcDNA3.1-LINC01186 plasmid, photograph magnification, $\mathrm{x} 50 .{ }^{*} \mathrm{P}<0.05$. 
A
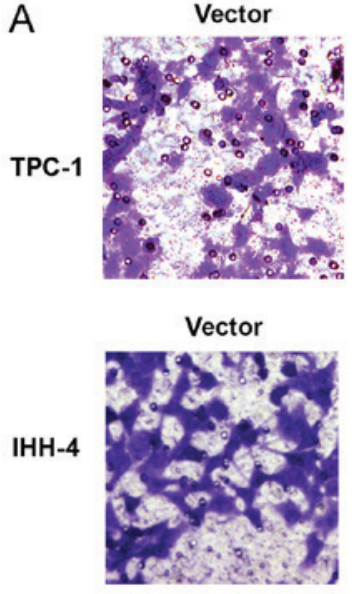

LINC01186

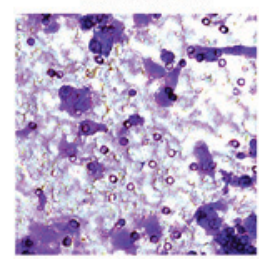

LINC01186

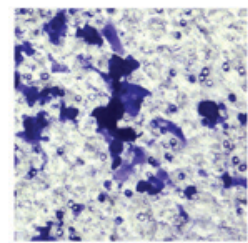

TPC-1

C

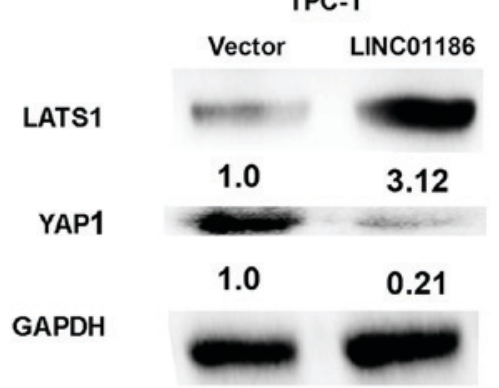

B $\square$ vector LINC01186

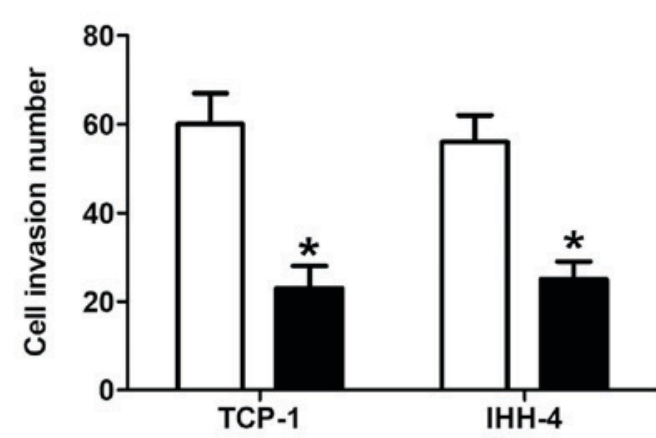

$\mathrm{D}$

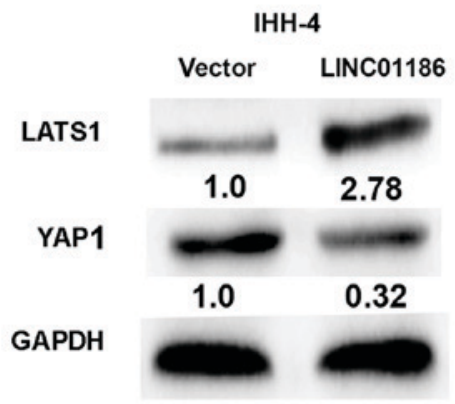

Figure 3. LINC01186 overexpression inhibited cell invasion and LATS1/YAP signaling in PTC cells. (A) Representative image and (B) quantification of Transwell assays used to detect cell invasion ability after TPC-1 and IHH-4 cells were transfected with pcDNA3.1-vector and pcDNA3.1-LINC01186 plasmid. Magnification, x200. The expression of LATS1 and YAP1 was detected using western blot after (C) TPC-1 and (D) IHH-4 cells were transfected with pcDNA3.1-vector and pcDNA3.1-LINC01186 plasmid. " $\mathrm{P}<0.05$. LATS1, large tumor suppressor kinase 1; YAP, YY1 associated protein 1.
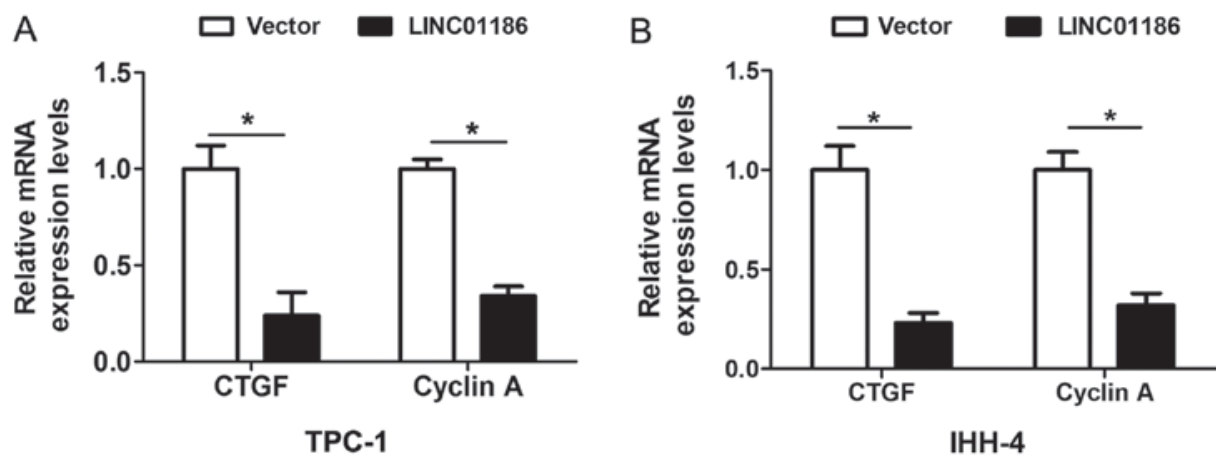

Figure 4. LINC01186 overexpression inhibited mRNA expression of CTGF and Cyclin A in PTC cells. (A) The expression of CTGF and Cyclin A was detected using RT-qPCR after TPC-1 cells were transfected with pcDNA3.1-vector and pcDNA3.1-LINC01186 plasmid. (B) The expression of CTGF and Cyclin A was detected using RT-qPCR after IHH-4 cells were transfected with pcDNA3.1-vector and pcDNA3.1-LINC01186 plasmid. "P<0.05.

we demonstrated that LINC01186 overexpression inhibited LATS1/YAP signaling pathway by downregulaing the YAP1 expression and increasing the LATS1 expression in PTC cells. Thus, our data suggested that LINC01186 expression closely related to LATS1/YAP in PTC. Of course, in the previous study, LINC01186 was found to suppress cell EMT process in lung cancer (10). In the future, we hope to investigate whether LINC01186 expression affects PTC cell EMT process by LATS1/YAP signaling pathway.

In conclusion, we found that LINC01186 was downregulated in PTC tissues and cells. LINC01186 acts as tumor suppressor to inhibit cell proliferation and invasion of PTC. Moreover, we demonstrated that LINC01186 overexpression inhibited LATS1/YAP signaling pathway by downregulating the YAP expression. Thus, our data suggested that LINC01186 may serve as a target for therapy in PTC.

\section{Acknowledgements}

Not applicable.

\section{Funding}

No funding was received. 


\section{Availability of data and materials}

The datasets used and/or analyzed in the present study are available from the corresponding author upon reasonable request.

\section{Authors' contributions}

NW and HD designed the study that led to the submission. CZ, $\mathrm{RG}$ and $\mathrm{YZ}$ performed functional experiments to investigate the roles of LINC01186 in PTC. NW and HD drafted the manuscript.

\section{Ethics approval and consent to participate}

The study protocol was approved by the Ethics Committee of Affiliated Hospital of Guizhou Medical University and written informed consent was obtained from all of patients.

\section{Patient consent for publication}

Not applicable.

\section{Competing interest}

The authors declare that they have no competing interests.

\section{References}

1. Sipos JA and Mazzaferri EL: Thyroid cancer epidemiology and prognostic variables. Clin Oncol (R Coll Radiol) 22: 395-404, 2010.

2. Lee YS, Lim YS, Lee JC, Wang SG, Kim IJ and Lee BJ: Clinical implication of the number of central lymph node metastasis in papillary thyroid carcinoma: Preliminary report. World J Surg 34: 2558-2563, 2010.

3. Siegel RL, Miller KD and Jemal A: Cancer statistics, 2017. CA Cancer J Clin 67: 7-30, 2017.

4. Roh JL, Park JY and Park CI: Total thyroidectomy plus neck dissection in differentiated papillary thyroid carcinoma patients: Pattern of nodal metastasis, morbidity, recurrence, and postoperative levels of serum parathyroid hormone. Ann Surg 245: 604-610, 2007.

5. Cheetham SW, Gruhl F, Mattick JS and Dinger ME: Long noncoding RNAs and the genetics of cancer. Br J Cancer 108: 2419-2425, 2013.

6. Murugan AK, Munirajan AK and Alzahrani AS: Long noncoding RNAs: Emerging players in thyroid cancer pathogenesis. Endocr Relat Cancer 25: R59-R82, 2018.

7. Zhou Q, Chen J, Feng J and Wang J: Long noncoding RNA PVT1 modulates thyroid cancer cell proliferation by recruiting EZH2 and regulating thyroid-stimulating hormone receptor (TSHR). Tumour Biol 37: 3105-3113, 2016.
8. Zhu H, Lv Z, An C, Shi M, Pan W, Zhou L, Yang W and Yang M: Onco-lncRNA HOTAIR and its functional genetic variants in papillary thyroid carcinoma. Sci Rep 6: 31969, 2016.

9. Wang C, Yan G, Zhang Y, Jia X and Bu P: Long non-coding RNA MEG3 suppresses migration and invasion of thyroid carcinoma by targeting of Rac1. Neoplasma 62: 541-549, 2015.

10. Hao Y, Yang X, Zhang D, Luo J and Chen R: Long noncoding RNA LINC01186, regulated by TGF-3/SMAD3, inhibits migration and invasion through Epithelial-Mesenchymal-Transition in lung cancer. Gene 608: 1-12, 2017.

11. Ribeiro FR, Meireles AM, Rocha AS and Teixeira MR: Conventional and molecular cytogenetics of human non-medullary thyroid carcinoma: Characterization of eight cell line models and review of the literature on clinical samples. BMC Cancer 8: 371, 2008.

12. Livak KJ and Schmittgen TD: Analysis of relative gene expression data using real-time quantitative PCR and the 2(-Delta Delta C(T)) method. Methods 25: 402-408, 2001.

13. Ugolini C, Borrelli N, Niccoli C, Elisei R, Viola D, Vitti P, Miccoli $\mathrm{P}$ and Basolo F: Role of YAP-1 in thyroid tumor progression and outcome. Appl Immunohistochem Mol Morphol 25: 581-585, 2017.

14. Liao T, Wen D, Ma B, Hu JQ, Qu N, Shi RL, Liu L, Guan Q, Li DS and Ji QH: Yes-associated protein 1 promotes papillary thyroid cancer cell proliferation by activating the ERK/MAPK signaling pathway. Oncotarget 8: 11719-11728, 2017.

15. Li Q, Shen W, Li X, Zhang L and Jin X: The lncRNA n340790 accelerates carcinogenesis of thyroid cancer by regulating miR-1254. Am J Transl Res 9: 2181-2194, 2017.

16. Li Z, Gao B, Hao S, Tian W, Chen Y, Wang L, Zhang X and Luo D: Knockdown of IncRNA-PANDAR suppresses the proliferation, cell cycle and promotes apoptosis in thyroid cancer cells. EXCLI J 16: 354-362, 2017.

17. Zhao JJ, Hao S, Wang LL, Hu CY, Zhang S, Guo LJ, Zhang G, Gao B, Jiang Y, Tian WG and Luo DL: Long non-coding RNA ANRIL promotes the invasion and metastasis of thyroid cancer cells through TGF- $\beta /$ Smad signaling pathway. Oncotarget 7: 57903-57918, 2016.

18. Liu K, Huang W, Yan DQ, Luo Q and Min X: Overexpression of long intergenic noncoding RNA LINC00312 inhibits the invasion and migration of thyroid cancer cells by down-regulating microRNA-197-3p. Biosci Rep 37: pii: BSR20170109, 2017.

19. Celano M, Mignogna C, Rosignolo F, Sponziello M, Iannone M, Lepore SM, Lombardo GE, Maggisano V, Verrienti A, Bulotta S, et al: Expression of YAP1 in aggressive thyroid cancer. Endocrine 59: 209-212, 2018.

20. Liu Z, Zeng W, Wang S, Zhao X, Guo Y, Yu P, Yin X, Liu C and Huang T: A potential role for the Hippo pathway protein, YAP, in controlling proliferation, cell cycle progression, and autophagy in BCPAP and KI thyroid papillary carcinoma cells. Am J Transl Res 9: 3212-3223, 2017.

This work is licensed under a Creative Commons Attribution-NonCommercial-NoDerivatives 4.0 International (CC BY-NC-ND 4.0) License. 